\title{
Vol. 70, No. 10
}

In the report "Association of State-Issued Mask Mandates and Allowing On-Premises Restaurant Dining with CountyLevel COVID-19 Case and Death Growth Rates - United States, March 1-December 31, 2020," on page 350, the third sentence in the first paragraph should have read, "Starting in April, 38 states and the District of Columbia (DC) issued mask mandates in 2020." 